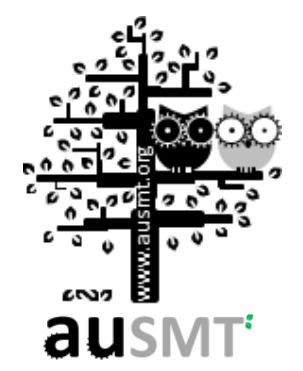

\title{
Applications of Intelligent Evolutionary Algorithms in Optimal Automation System Design
}

\section{Tung-Kuan Liu and Jyh-Horng Chou*}

Department of Mechanical and Automation Engineering, National Kaohsiung First University of Science and Technology, Taiwan (Received 31 March 2011; Published on line 1 September 2011)

*Corresponding author: choujh@nkfust.edu.tw DOI: 10.5875/ausmt.v1i1.100

\begin{abstract}
This paper proposes an intelligent evolutionary algorithm that can be applied in the design of optimal automation systems, and employs a multimodal six-bar mechanism optimization design, job shop production scheduling for the fishing equipment industry, and dynamic real-time production scheduling system design cases to show how the technique developed in this paper is highly effective at resolving optimal automation system design problems. Major breakthroughs in artificial intelligence continue to be made in the wake of advanced information technology developments, and the field of intelligent evolutionary algorithms has attracted a particularly large amount of attention from researchers and users in the artificial intelligence community. The successful optimization of automation system design requires interdisciplinary integration, and further requires the use of actual cases, verification, and improvement to ensure implementation in real-world applications.
\end{abstract}

Keywords: Intelligent Evolutionary Algorithms; Optimized Automation System Design; multimodal six-bar mechanism; artificial intelligence.

\section{Introduction}

The machinery, manufacturing, processing, metals, electronics, and electrical machinery industries have long needed to resolve problems associated with linkage mechanisms and production scheduling in order to overcome bottlenecks. Linkage mechanisms offer advantages such as structural simplicity, stable action, and lower costs, and are widely applied in manufacturing plants. The design of linkage mechanisms usually considers only a specific path of movement with a composite structure. However, this limits the path of motion, which restricts processing conditions and reduces production flexibility. This paper proposes a multimodal linkage mechanism design that can enable a mechanism to adjust certain parts or angles of rotation during operation, enabling it to reach several processing locations within its processing scope. Furthermore, production scheduling is an important method of achieving cost control and on-time shipments. While practical scheduling is usually conducted on the basis of experts' knowledge and experience, in the most complex and variable operating environments, experts may commit a tremendous amount of time and effort without meeting actual needs, causing losses of time and funds in the process. This paper proposes that the intelligent Taguchi genetic algorithm be used to resolve optimal multimodal six-bar mechanism design, fishing equipment industry job shop production scheduling, and dynamic real-time production scheduling system design problems. The intelligent Taguchi genetic algorithm chiefly consists of a conventional genetic algorithm [1] and the Taguchi method [2, 3]. The Taguchi method employs the reproduction operations of a conventional genetic algorithm, and can achieve an optimal offsprings after many instances of testing and selection of highly-influential genes. By increasing stability and convergence speed, this method can successfully resolve difficulties faced in the design of optimal automation systems. 


\section{Intelligent Genetic Algorithms}

This chapter provides a detailed explanation of the design of chromosomes in intelligent genetic algorithms, calculation of fitness values, and genetic algorithm methods and steps.

\section{Description of intelligent genetic algorithms}

This paper proposes a new and stable method termed the intelligent genetic algorithm. This method applies the Taguchi method to the reproduction stage of a conventional genetic algorithm [4-6], and employs a two-level orthogonal array at the time of reproduction. The use of an orthogonal array can minimize the number of trials needed, yielding more reliable factor characteristics. In addition, the signal-to-noise ratio is calculated in order to assess the ability of each factor to find highly influential genes and thereby produce optimal offsprings. Furthermore, this method preserves the fittest individuals after each round of calculations; when the number of individuals is equal to the number of populations, the next round of calculations is performed using the new population. The following is an

Tung-Kuan Liu received the B.S. degree in Mechanical Engineering from National Akita University, Japan, in 1992 and the M.S. and Ph.D. degrees in Mechanical Engineering and Information Science from National Tohoku University, Japan, in 1994 and 1997, respectively. He is currently an Associate Professor of Mechanical and Automation Engineering Department at National Kaohsiung First University of Science and Technology, Taiwan. From 1997 to 1999, he was a Senior Manager of Institute of Information Industry, Taiwan. From 1999 to 2002, he was an Assistant Professor of Department of Marketing and Distribution Management at National Kaohsiung First University of Science and Technology, Taiwan. His research and teaching interests include artificial intelligence, applications of multi-objective optimization genetic algorithms, and integrated manufacturing and business systems. Two of his publications in IEEE Transactions on Evolutionary Computation (vol. 8, pp. 365-377, 2004) and IEEE Transactions on Neural Networks (vol. 17, pp. 69-80, 2006) have been selected as the "Highly Cited Papers" by ISI Essential Science Indicators since 2011.

Jyh-Horng Chou received the B.S. and M.S. degrees in Engineering Science from National Cheng-Kung University, Taiwan, in 1981 and 1983, respectively, and the Ph.D. degree in Mechatronic Engineering from National Sun Yat-Sen University, Taiwan, in 1988. He is currently a Professor of National Kaohsiung First University of Science and Technology, where he has been served as the Vice President and the Acting President from 2006 to 2010. He has coauthored three books, published more than 218 refereed journal papers and 213 conference papers, in which two journal papers published in the IEEE Transactions have been selected to be the Highly Cited Papers by ISI Essential Science Indicators. His research and teaching interests include intelligent systems and control, computational intelligence and methods, automation technology, robust control, and quality engineering. Dr. Chou has been elected as the Most Cited Scientists in Engineering Field by ISI Essential Science Indicators. He also received many awards including the Excellent Research Award from the National Science Council of Taiwan, the 2004 Excellent Project Outcome Award of the Educational Promotion Project on the Integrated Manufacturing and e-Commerce Technology from the Ministry of Education, Taiwan, the 2007 Distinguished Electrical Engineering Professor Award from the Chinese Institute of Electrical Engineering, Taiwan, the 2007 Excellent Educator Award from the Ministry of Education, Taiwan, the 2010 Distinguished Engineering Professor Award from the Chinese Institute of Engineers, Kaohsiung Chapter, Taiwan, and the 2010 Distinguished Engineering Professor Award from the Chinese Society of Mechanical Engineers, Taiwan. introduction to the steps used by an intelligent genetic algorithm and a description of how the Taguchi method can be applied to a conventional genetic algorithm:

Encoding:

The encoding method used in this paper employs chromosome genes to indicate real number variables and parts. The real number variable encoding can be found in the later section entitled "Chromosome design" for a detailed account; the encoding of parts is as follows: The first part is expressed as "1," and the second part is expressed as "2"; other parts are denoted in this manner $[2,4,7]$. For example, assuming that a certain work piece is composed of three parts, and each part consists of three processes, the processing sequence and machine processing time would be as shown in Tables 1 and 2 .

Table 1. Parts processing sequence

\begin{tabular}{|l|l|l|l|}
\hline & Process 1 & Process 2 & Process 3 \\
\hline Part 1 & 1 & 2 & 3 \\
\hline Part 2 & 2 & 1 & 3 \\
\hline Part 3 & 2 & 3 & 1 \\
\hline
\end{tabular}

Table 2. Machine processing time

\begin{tabular}{|l|l|l|l|}
\hline & Part 1 & Part 2 & Part 3 \\
\hline Machine 1 & 2 & 5 & 3 \\
\hline Machine 2 & 4 & 2 & 1 \\
\hline Machine 3 & 3 & 2 & 4 \\
\hline
\end{tabular}

Here, the chromosomal OP gene is distributed as $O P=\{1,1,1,2,2,2,3,3,3\} \quad$, where the first gene-"1"-in the OP array represents the first processing procedure of the first part, the processing machine is the first machine, and the processing time is 2 units. The second gene-"1"-represents the second processing procedure for the first part, where the processing machine is the second machine and the processing time is 4 units. The third gene-"1"-represents the third processing procedure for the first part, where the processing machine is the third machine and the processing time is 3 units. The fourth gene represents the first processing procedure for the second part, where the processing machine is the second machine and processing time is 2 units. The fifth gene represents the second processing procedure for the second part, where the processing machine is the first machine and the processing time is 5 units, and so on for the remaining genes.

\section{Initialization:}

Produces a chromosome representing $N$ parts and $Q$ processes, and randomly substitutes gene loci. 
Selection:

This paper employs the roulette wheel selection method [8, 9]. In the genetic algorithm, the fitness value is used to judge the fitness of each chromosome; the fitness of each chromosome in the population is the standard for the selection or rejection of that chromosome.

The fitness of each chromosome is used to determine its size on the roulette wheel; the greater the fitness, the larger the area it occupies on the roulette wheel. Conversely, the lower the fitness, the smaller the area on the roulette wheel. Here, area on the roulette wheel expresses the probability that a chromosome will be selected; during selection, two parent generation genes cross over.

\section{Set Partition Crossover:}

To ensure that conflict between genes does not occur after the crossover operation, this paper employs the set partition crossover method [10] to perform crossover, and tests the chromosomes in conjunction with the production of an orthogonal array; the set partition crossover steps were as follows:

Step 1: Assuming $i=0$, generate an integer corresponding to the number of parts and save in the $S$ set.

Step 2: Divide the $S$ set into the two subsets $S_{1}$ and $S_{2}$, where $S_{1} \cap S_{2}=\varphi$ and $S=S_{1} \cup S_{2}|S|=\left|S_{1}\right|+\left|S_{2}\right|$.

Step 3: When the first element in the $S$ set corresponds to the orthogonal array element "1", place that element in the $S_{1}$ set; if it corresponds to the orthogonal array element "2", place it in the $S_{2}$ set. Use this principle to generate the $S_{1}$ and $S_{2}$ sets.

Step 4: Use the $i^{\text {th }}$ gene in the two parent generations $R_{1} i^{\text {th }}$ and $R_{2} i^{\text {th }}$ as the $i^{\text {th }}$ group corresponding to the $S_{1}$ and $S_{2}$ sets, and so on.

Step 5: If a gene corresponding to the $i^{\text {th }}$ gene in the first parent generation $R_{1} i^{\text {th }}$ can be found in set $S_{1}$, place that gene in a new chromosome; if a corresponding gene cannot be found, ignore that gene.

Step 6: If a gene corresponding to the $i^{\text {th }}$ gene in the second parent generation $R_{2} i^{\text {th }}$ can be found in set $S_{2}$, place that gene in a new chromosome; if a corresponding gene cannot be found, ignore that gene.

Step 7: if $i<$ chromosome length, then $i=i+1$, repeat steps 4-6.

Orthogonal array selection in the Taguchi method is chiefly determined by the number of individuals with the most parameters and the lowest number of trials. We used a two-level orthogonal array in this paper and established a two-level orthogonal array with $N$ factors, where each factor corresponded to an element in set $S$. In $L_{n}\left(2^{n-1}\right), n-1$ is the number of rows, and $\mathrm{n}$ is the number of trials. When $N<n-1$, factors in the orthogonal array are used starting with the first factor, and the unused factors can be ignored.

The next step was to combine the selected orthogonal array with the set partition crossover used in this paper. The elements in the set $S$ were first used as the factors in the orthogonal array. If any factor corresponds to the element "1" in the orthogonal array, that element is placed in set $S_{1}$; and if the factor corresponds to the element "2" in the orthogonal array, that element is placed in set $S_{2}$. When set $S$ becomes an empty set, reproduction steps 4 to 7 are implemented. This process is continued until the number of chromosomes is consistent with the number of trials in the orthogonal array, and a trial is performed; the optimal results of the trial are chosen as an offspring generation. For example, a certain work piece is composed of six parts, and each part has two processes. The $L_{8}\left(2^{7}\right)$ orthogonal array shown in Table 3 is selected. Here the values $S=\{1,2,3,4,5,6\}$ correspond to the orthogonal array factors A, B, C, D, E, and F. The method of generating $S_{1}$ and $S_{2}$ and the new chromosome production steps are illustrated in Figure 1.

Table 3. $L_{8}\left(2^{7}\right)$ orthogonal array [11].

\begin{tabular}{|l|l|l|l|l|l|l|l|}
\hline \multirow{3}{*}{ Number of trials } & \multicolumn{7}{|l|}{ Trial factors } \\
\cline { 2 - 8 } & A & B & C & D & E & F & G \\
\cline { 2 - 8 } & 1 & 2 & 3 & 4 & 5 & 6 & 7 \\
\hline 1 & 1 & 1 & 1 & 1 & 1 & 1 & 1 \\
\hline 2 & 1 & 1 & 1 & 2 & 2 & 2 & 2 \\
\hline 3 & 1 & 2 & 2 & 1 & 1 & 2 & 2 \\
\hline 4 & 1 & 2 & 2 & 2 & 2 & 1 & 1 \\
\hline 5 & 2 & 1 & 2 & 1 & 2 & 1 & 2 \\
\hline 6 & 2 & 1 & 2 & 2 & 1 & 2 & 1 \\
\hline 7 & 2 & 2 & 1 & 1 & 2 & 2 & 1 \\
\hline 8 & 2 & 2 & 1 & 2 & 1 & 1 & 2 \\
\hline
\end{tabular}

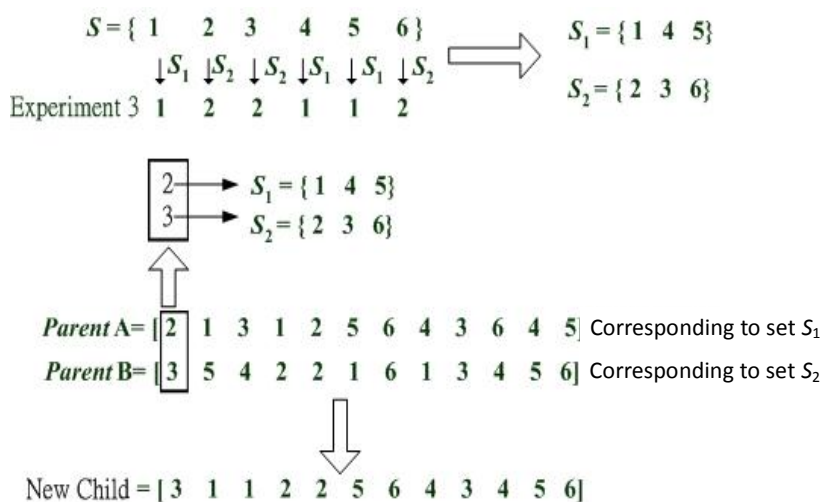

Figure 1. Schematic diagram of reproduction 
After completing the trial, the signal-to-noise ratio $(S / N$ ratio) is calculated to determine the dispersion of each target function following the trial. This allows the mutual influence of different factors to be analyzed. If a larger target function is more desirable, then $Z_{t}=\left(y_{t}\right)^{2}$, and if a smaller target function is more desirable, then $Z_{t}=\left(1 / y_{t}\right)^{2}$. Assuming that $y_{t}$ is the target function following the trial, its value will be expressed by following Equation:

$$
S / N_{j k}=\sum_{t=1}^{n} Z_{t} \quad(j=1,2, \ldots ., N)
$$

$j$ : influencing factors

$k$ : number of levels

$t$ : number of trials

After analysis of the $S / N$ ratio yields the most important factors, these factors are used to find the best combination after a Taguchi trial. When $\left(S / N_{j 1}>S / N_{j 2}\right)$ indicates that this factor's first-level factor has the greatest influence, this factor is placed in set $S_{1}$, otherwise the second-level factor has the greatest influence, and this factor is placed in set $S_{2}$. This sequential comparison process yields the best combination. This combination is then used to generate sets $S_{1}$ and $S_{2}$ and perform reproduction steps 4 to 7 ; the resulting chromosomes belong to the second offspring generation.

Mutation:

We employed the neighbor search mutation (NSM) method $[7,8]$ to perform mutation in this study. For instance, in a problem with four parts and four machines, three randomly selected genes yield $P_{2}^{3}=6$ combinations. After calculating the value choices corresponding to each combination, fit chromosomes were selected for offspring generation after mutation (Figure 2).

\section{Elimination:}

Calculation of fitness of all chromosomes and ranking, selection of the fittest chromosome as the new population for the next round of calculations.

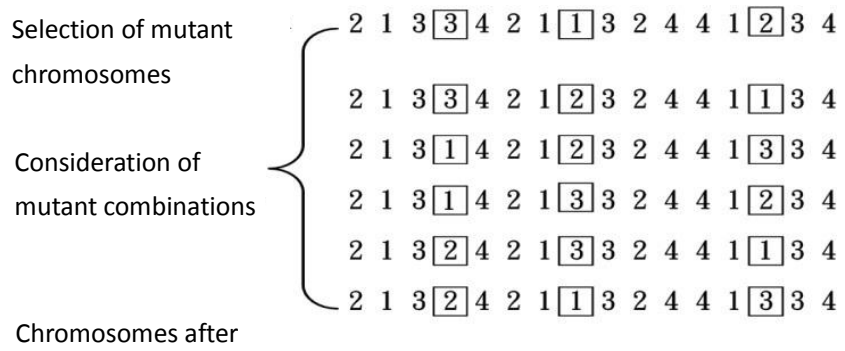

mutation

\begin{tabular}{lllllllllllll|lll}
2 & 1 & 3 & 2 & 4 & 2 & 1 & 3 & 3 & 2 & 4 & 4 & 1 & 2 & 3 & 4
\end{tabular}

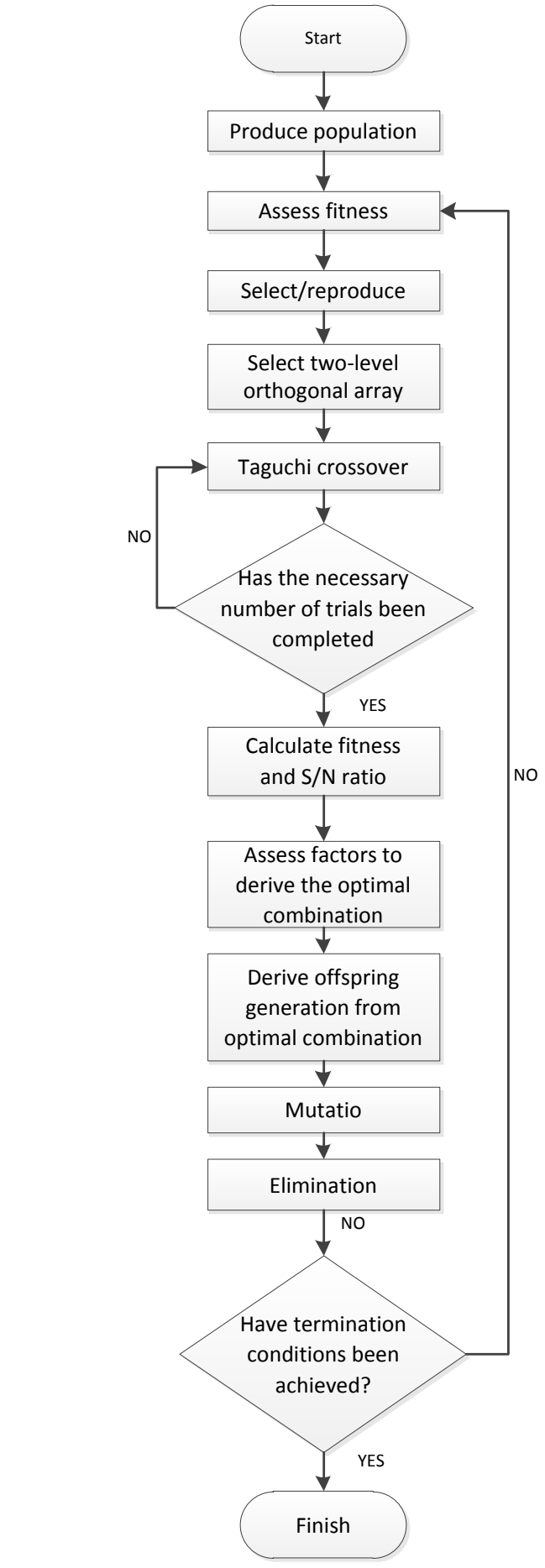

Figure 3. Algorithm flowchart.

Calculation steps using an intelligent Taguchi genetic algorithm

An intelligent Taguchi genetic algorithm combines a conventional genetic algorithm with the Taguchi method and then adds reproduction. Combining reproduction with the Taguchi method allows for the most effective genes after computation to be selected using the genetic algorithm. The following are calculation steps employing an intelligent Taguchi genetic algorithm (see flowchart in Figure 3):

Figure 2. Schematic diagram of mutation. 
Step 1: Parameter settings

Input: parent generation number, reproduction rate, mutation rate, algorithm generation number.

Output: optimal total schedule, chromosomes, scheduling.

Step 2: Chromosome encoding, calculation of fitness, initialization.

Step 3: Establish a roulette wheel and select two parent generations for reproduction.

Step 4: Select a two-level orthogonal array.

Step 5: Perform crossover.

Step 6: Select the optimal total schedule results using orthogonal array trials as the first offspring generation.

Step 7: Calculate the signal to noise ratio, assess the efficiency of each factor efficiency, and find the best combination.

Step 8: Repeatedly perform reproduction calculations using the best combination to produce the second offspring generation.

Step 9: Perform mutation.

Step 10: Determine whether the necessary number of offspring generations has been produced; if not, repeat step 5 .

Step 11: Perform elimination.

Step 12: Display final computation results.

In scheduling problems, each gene represents a part of the process, and fitness calculation must comply with two restrictive conditions:

(1) In the case of parts, the start time for the next process involving the same part may not be as early as the completion time for the same part's previous process.

(2) With regard to machinery, the same machine may not process two or more parts at the same time. This paper does not consider whether or not the machine is capable of processing other parts during idle time, and parts are placed in the machine and processed in sequence. This study combines a conventional genetic algorithm with the Taguchi method, and employs the theory of the Taguchi method to increase the calculation convergence rate and reduce computation time.

\section{Multiobjective Optimization}

Multiobjective optimization is a mathematical programming method that can clearly and simultaneously consider multiple decision-making objectives. Its goal is to help decision-makers find an optimal action plan under circumstances of limited resources and conflicts between different objectives.

In order to solve multiobjective optimization problems, the problems can first be classified on the basis of difficulty as P (i.e., polynomial), and NP-hard (non-polynomial hard) types. Scheduling problems can similarly be classified as two types:

(1) Non-polynomial time algorithm solvable problems (NP problems).

(2) Polynomial time algorithm solvable problems.

The following methods are commonly used to solve multiobjective programming problems:

(1) Weighting method: The weighting method assigns a fixed weighting to each individual objective, and employs the weighting concept to convert a multiobjective function into overall objective function, and then further derive a single optimal solution.

(2) Pareto optimal solution: The Pareto optimal solution method does not assign a fixed waiting to individual objectives, nor does it assign preferences to individual objections. The weightings of different objectives are generated using a randomized method during the derivation process, and then the algorithm is used to search for the optimal solution among the variable weightings.

(3) Fuzzy goal programming: In contrast to conventional goal programming and utility functions, fuzzy programming does not require precise objective values to be supplied, and each individual objective can be considered to possess different characteristics; as a result, fuzzy satisfaction can be used to assess the degree of advantage of each individual objective.

\section{Optimized Automation System Design}

This chapter will investigate the applications of intelligent evolutionary algorithm technology on optimal automation systems design in the three cases of multimodal six-bar mechanism optimization design, fishing equipment industry job shop production scheduling, and dynamic real-time production scheduling system design.

\section{The multimodal six-bar mechanism optimization design case}

In recent years, synthetic research on adaptable mechanisms has employed many cases to perform planning of complex motion paths $[12,13]$. 
The optimization method is free from both the restriction of precise location analysis in conventional graphical methods, and can also derive more precise synthetic mechanisms, simultaneously consider path of motion and mechanism synthesis, and perform dynamic analysis of linkage mechanisms encompassing the properties of speed, acceleration, torque, etc. $[9,14,15]$.

\subsection{Synthesis of mechanism and analysis}

The following steps can be used to perform the synthesis and analysis of a mechanism in the design process (see flowchart in Figure 4) $[16,17]$.

The mechanisms must be able to complete processing of different processing injection points within the processing scope and also generate a path of motion (see Figure 5) [18].

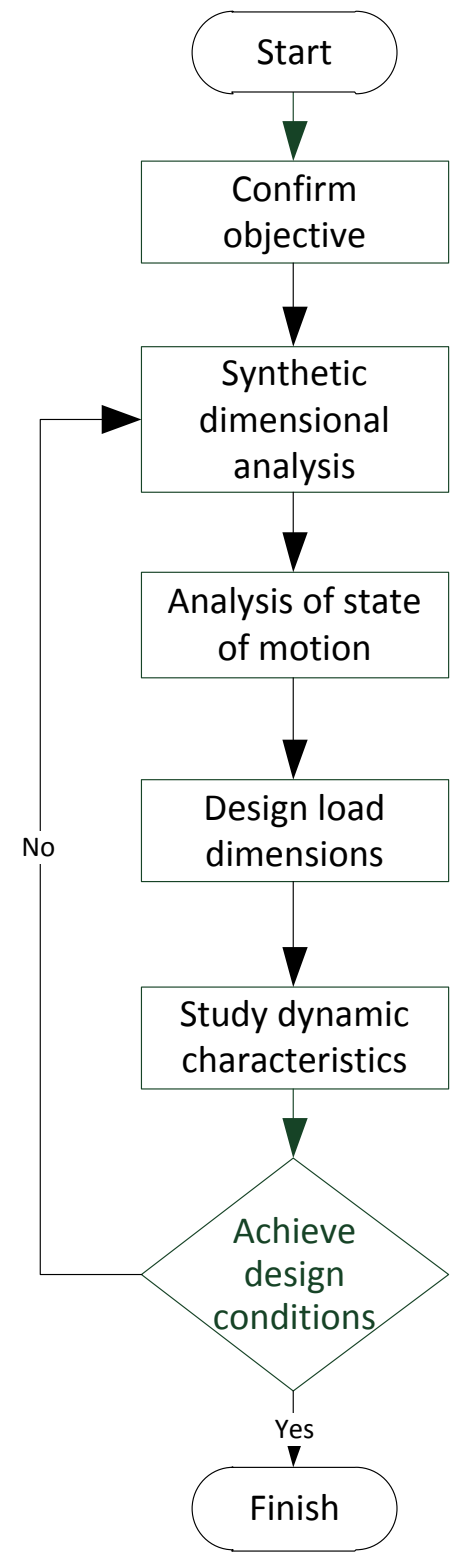

Figure 4. Mechanism analysis flowchart.

Figure 4. Mww.ausmt.org

Copyright (C) 2011 International Journal of Automation and Smart Technology
The design employed in this paper relies on adjustment of rod length $\overline{E F}$ in conjunction with different angles of movement to complete processing of different processing injection points within the processing scope and simulate the link path of motion (see Figure 6).

This case uses the four extreme points within the maximum processing scope as the casting points of the preliminary synthetic multimodal six-bar structure. Here points $A, B, C$, and $D$ represent the locations of the four extreme points (see Figure 6).

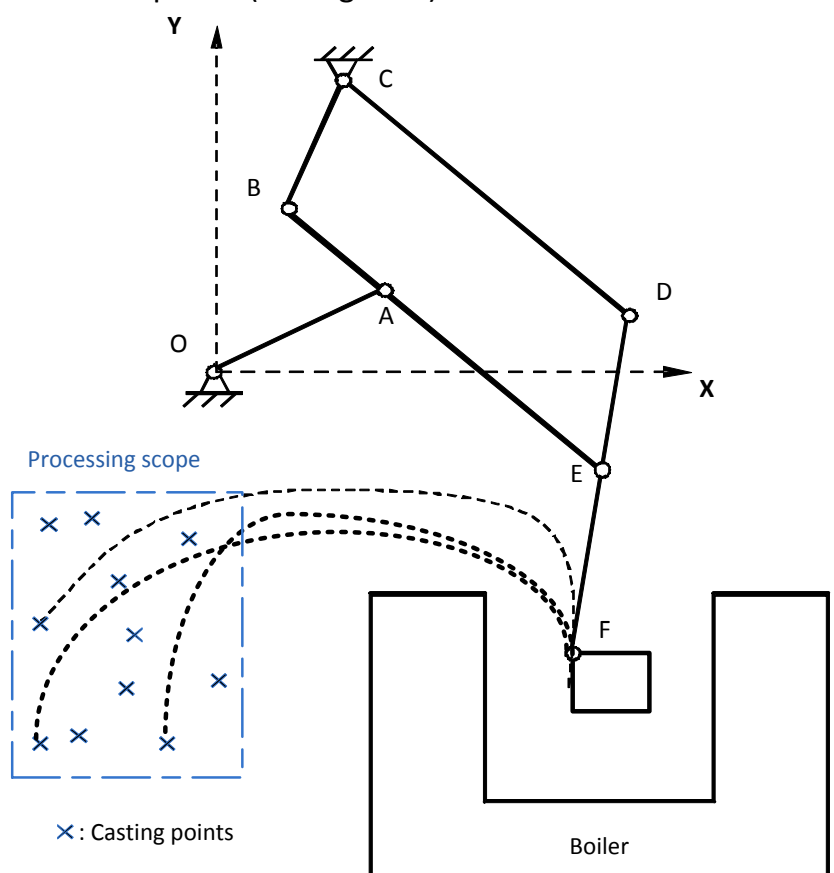

Figure 5. Schematic diagram of six-bar processing scope and path of motion.

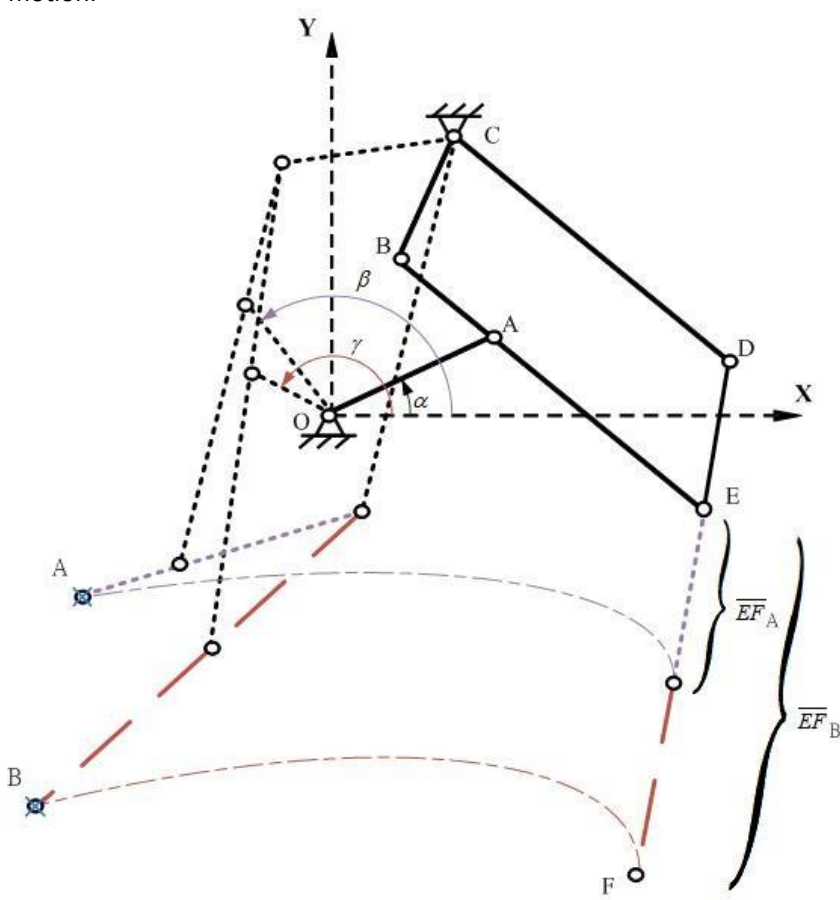

Figure 6. Schematic diagram of action of multimodal six-bar mechanism. 
This case uses the four extreme points within the maximum processing scope as the casting points of the preliminary synthetic multimodal six-bar structure. Here points $A, B, C$, and $D$ represent the locations of the four extreme points (see Figure 7).

When an intelligent evolutionary algorithm technology was used to design basic linkage structure (see the red portion of Figure 7), the rod length $\overline{E F}$ and operating angle were adjusted to derive an optimal mechanism design with processing injection points distributed throughout the processing scope. This allowed the rod length $\overline{E F}$ and operating angle needed for the casting points to be found, which in turn enabled the rod length $\overline{E F}$ and operating angle to be adjusted to accommodate the casting locations (see Figure 8).

\subsection{Chromosome design}

This case employed real number encoding to initialize the gene population in the chromosomes. In order to accommodate optimization of multiple casting paths, this paper employed rod lengths $r_{1}$ to $r_{6}$ and $C$ points as the fixed genes in the chromosomes, and employed numbers of casting points to generate several different angles of movement and $\overline{E F}$ rod lengths, ensuring that each processing point had its own individual angles of movement and $r_{7}$ rod lengths (see Figure 9) [19-21]. Consequently, a chromosome (Figure 10) contained eight fixed genes and $3 \times p$ floating genes, where $p$ indicated the number of casting points at each search (see Equation (2)).

$$
X_{m}^{n}=\left[x_{1}^{n}, x_{2}^{n}, \ldots, x_{m}^{n}\right], \forall_{x} \in R
$$

and

$$
m=t+(p \times 3)
$$

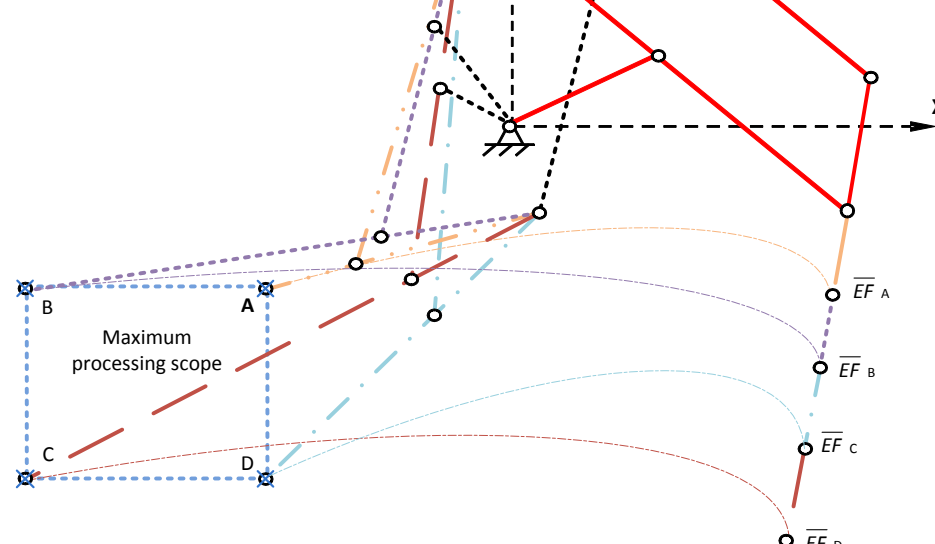

where,

$n$ : initial number of chromosomes,

$m$ : number of genes,

$t$ : number of fixed genes,

$p$ : number of casting points.

Figure 7. Schematic diagram of maximum multimodal six-ba processing scope.

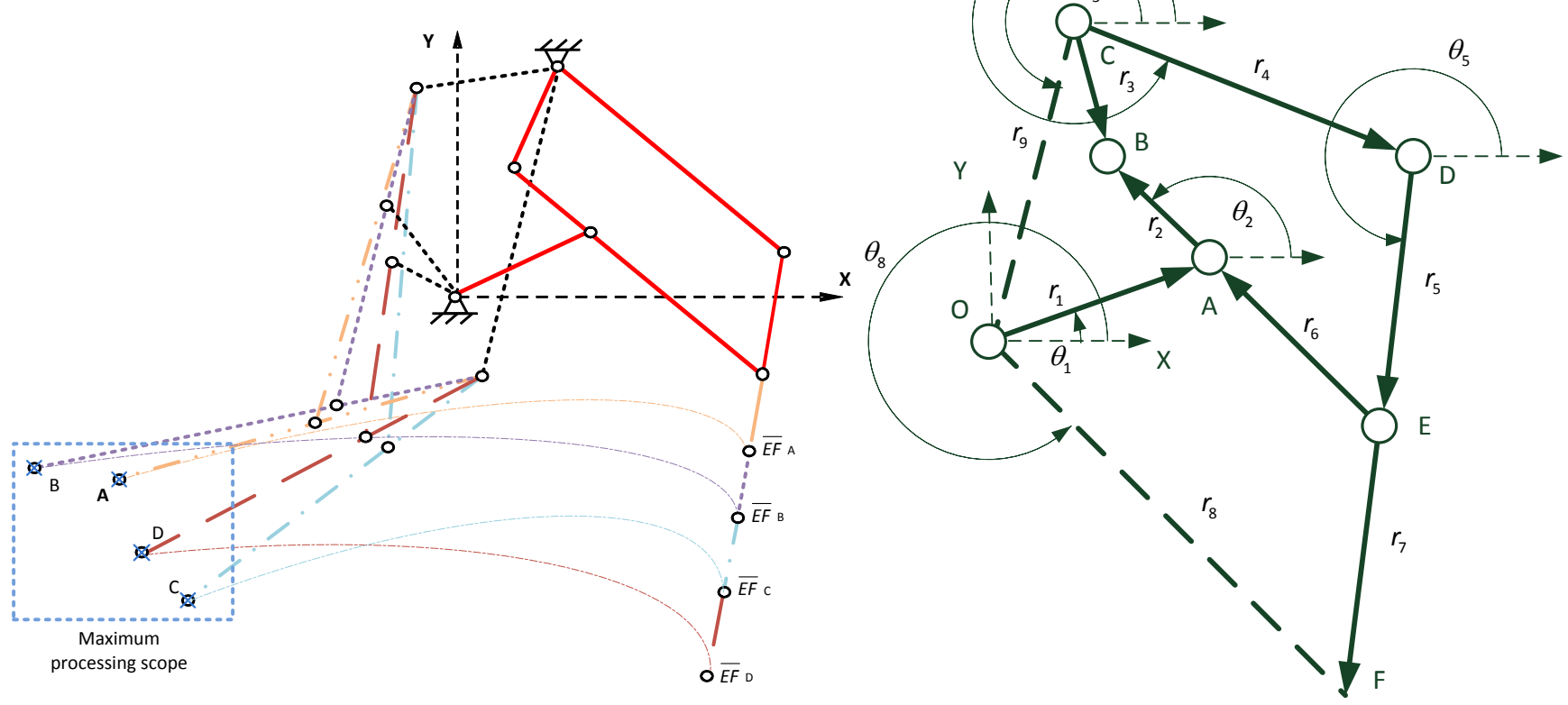

Figure 8. Schematic diagram of multimodal six-bar structure in

Figure 9. Six-bar vector circuit diagram. sub-processing scope. 


\section{Multimodal six-bar basic structure}

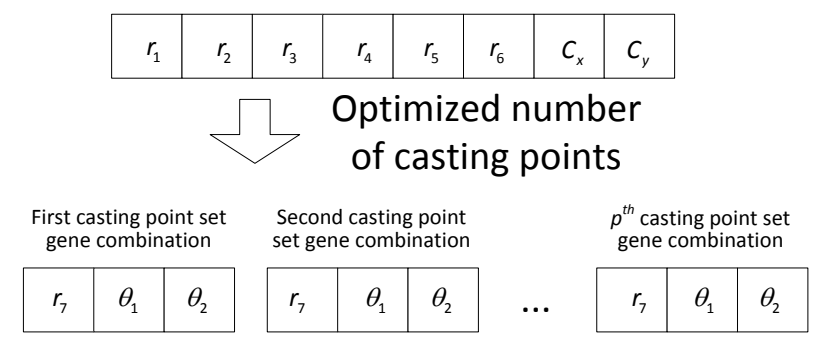

Figure 10. Schematic diagram of multimodal six-bar gene encoding

The algorithmic steps are as shown below:

Step 1: random generation of $\beta$ values, where $\beta \in[0,1]$. Step 2: use this Equation, $x_{i}=l_{i}+\beta\left(u_{i}-l_{i}\right)$, to find the initial chromosome gene population. Here $l_{i}$ and $u_{i}$ are the upper and lower limits of the variables.

Step 3: Repeat steps 1 to 2 until $n$ chromosomes have been completed.

The multimodal six-bar structure rod length $\overline{E F}$ and mechanism operating angles in the chromosomes' casting points are as shown below:

$u: u^{\text {th }}$ casting point,

$E F \_u$ : rod length $\overline{E F}$ of the $u^{\text {th }}$ casting point

$S \_u$ : initial link angle of the $u^{\text {th }}$ casting point, and

$E_{-} u$ : final link angle of the $u^{\text {th }}$ casting point.

\subsection{Fitness design}

With regard to the chromosome fitness calculation method, the largest error resulting from the path of motion of each group of casting paths is taken as the casting path error. The casting path errors of all groups are added to derive the chromosome's fitness.

When using chromosomes to calculate the link path of motion and boiler collisions, the chromosome fitness plus punishment value will ensure that a chromosome's characteristics are preserved in the chromosome population, and the objective function is as shown in Equation (4):

$$
D=\sum_{i=1}^{p} \min \left(d_{i}\right)
$$

And

$$
d_{i}=\left\{\begin{array}{cc}
P_{V}, & d_{i} \text { overlap } O_{b s} \\
\max _{j}\left(Q_{i, j}\right), & \text { otherwise }
\end{array}\right.
$$

$$
\begin{aligned}
& Q_{i, j}=\sqrt{\left(\overline{X_{i, j}}-X_{i, j}\right)^{2}+\left(\overline{Y_{i, j}}-Y_{i, j}\right)^{2}} \\
& Q_{i, j}=\left\{\begin{array}{lc}
0, & Q_{i, j}<\varepsilon \\
Q_{i, j}, & \text { otherwise }
\end{array}\right.
\end{aligned}
$$

Where:

$p$ : searched for number of casting points,

$D$ : total error of each group of casting paths,

$Q_{i, j}$ : the error produced by the processing points in each group of casting paths,

$d_{i}: i^{\text {th }}$ casting path,

$\varepsilon$ : acceptable error range,

$P_{v}$ : punishment value,

$O_{b s}:$ boiler location,

$\left(\bar{X}_{i, j}, \bar{Y}_{i, j}\right)$ : precise points $j$ through which a link to the $i^{\text {th }}$ casting path must pass,

$\left(X_{i, j}, Y_{i, j}\right)$ : actual corresponding points when a link to the $i^{\text {th }}$ casting path passes precise points $j$.

\subsection{Results and discussions}

This paper performed two multimodal six-bar optimization designs. In order to obtain more results from the optimization algorithm, this study repeated the first optimized synthetic design experiment 10 times in an effort to achieve more optimal solutions by conducting a relatively large number of experiments. The study then selected the three most representative experimental results and calculated the optimal processing design within the relevant sub-range. The sub-range processing optimization algorithm experiment was performed only once.

The second optimization synthesis experiment used the rod lengths $r_{1}$ to $r_{6}$ and $C$ points from the first optimization synthesis experiment as a basic structure, and distributed 18 casting points through the maximum processing scope so that the 18 points were evenly distributed through the processing scope and could serve as sub-range processing casting points. In addition, this case designated the 18 casting points as type I casting points, type II casting points, and type III casting points. There were eight type I casting points, six type II casting points, and four type III casting points. The chromosome length was adjusted each time the algorithm searched for the number of casting points, ensuring that the algorithm could perform optimal synthesis of several casting points. 


\subsection{Contribution}

(1) Use of the genetic algorithm increased the accuracy of the solution of the linkage mechanism synthesis problem.

(2) Application of the genetic algorithm accelerated the design of a six-bar linkage mechanism which was actual used in casting work (see Figures 11).

Linkage synthesis is not limited to Grashof mechanisms. Since the scope of other papers has been limited to Grashof mechanisms, this is a big step forward.

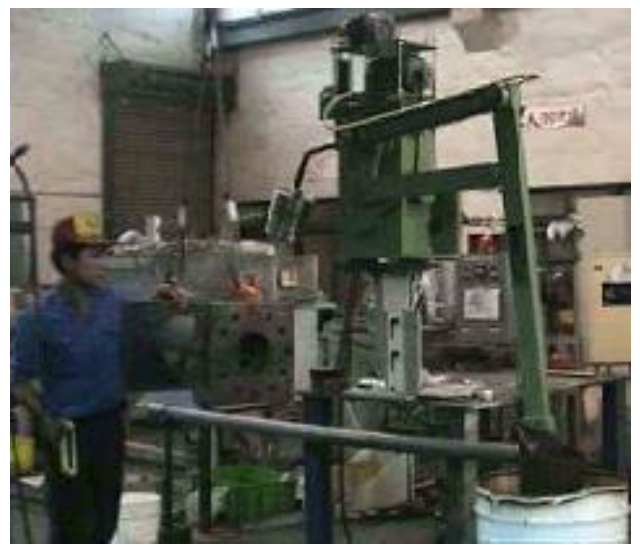

(a)

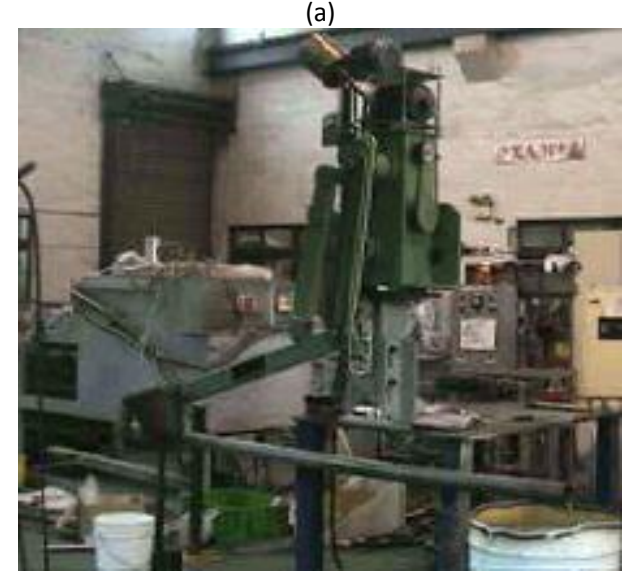

(b)

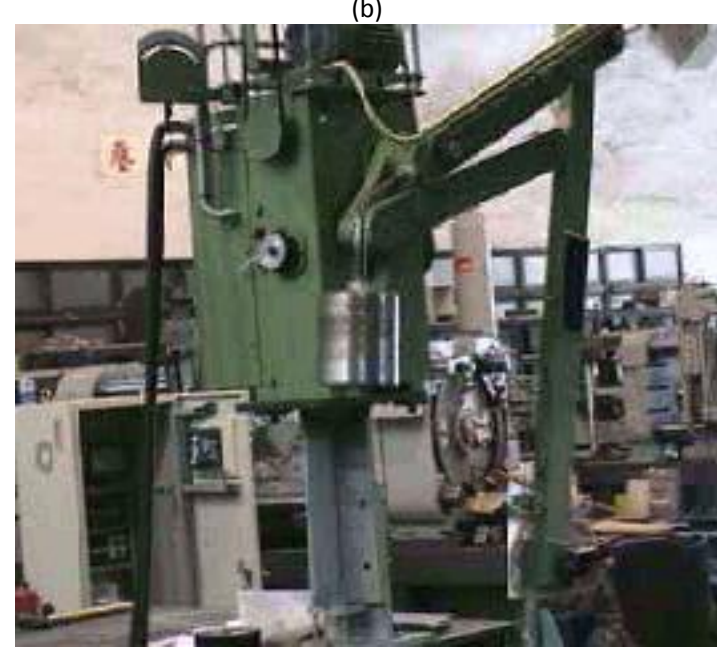

(c)

Figure 11. Six-bar linkage machine.
2. A fishing equipment industry job shop production scheduling case

Production scheduling in the fishing equipment industry currently involves the use of conventional determinative methods, in which scheduling personnel rely on their experience to perform scheduling. This approach consumes large amounts working time, greatly limits understanding space, and cannot be used to derive a globally optimal production scheduling solution. Because of this, numerous studies have used genetic algorithms to resolve this type of scheduling problem [22].

\subsection{Production processes in the fishing equipment} industry

This paper performs production scheduling for the bottleneck processes of filament pulling and net weaving. After considering task sequence, production scheduling must consider one or more performance assessment guidelines, which can be used to assess the effectiveness of scheduling order. Assessment guidelines can generally be classified as the four major types of time-related, product and machinery-related, delivery time-related, and cost-related guidelines (Table 4) [23]. Fishing equipment industry production processes are as shown in Figure 12.

Table 4. Production scheduling performance assessment guidelines.
\begin{tabular}{|l|l|}
\hline Time-related assessment & $\begin{array}{l}\text { Average processing time } \\
\text { Maximum completion time } \\
\text { Average waiting time }\end{array}$ \\
\hline $\begin{array}{l}\text { Product and machinery-related } \\
\text { assessment }\end{array}$ & $\begin{array}{l}\text { Average quantity in product } \\
\text { Average number awaiting working } \\
\text { Average machinery use rate }\end{array}$ \\
\hline $\begin{array}{l}\text { Delivery date-related } \\
\text { assessment }\end{array}$ & $\begin{array}{l}\text { Average differential time } \\
\text { Average delay time } \\
\text { Number of delayed jobs }\end{array}$ \\
\hline Cost-related assessment & $\begin{array}{l}\text { Total processing cost } \\
\text { Average waiting cost } \\
\text { Product inventory cost }\end{array}$ \\
\hline
\end{tabular}

\subsection{Chromosome design}

The chromosomes used in this case are expressed as shown in Table 5 . The chromosomes contain $N$ genes, which represent the allocation of machine resources when there are $N$ work pieces. Each gene represents each scheduled work piece, and the gene locations represent the order in which machine resources are used. Each chromosome represents a feasible scheduling result.

Table 5. shows how the total number of work pieces is arranged as a chromosome.

\begin{tabular}{|l|l|l|l|l|l|l|l|l|l|l|l|l|}
\hline 1 & 2 & 3 & 4 & 5 & 6 & 7 & 8 & 9 & 10 & 11 & $\ldots$ & $\mathrm{N}$ \\
\hline
\end{tabular}




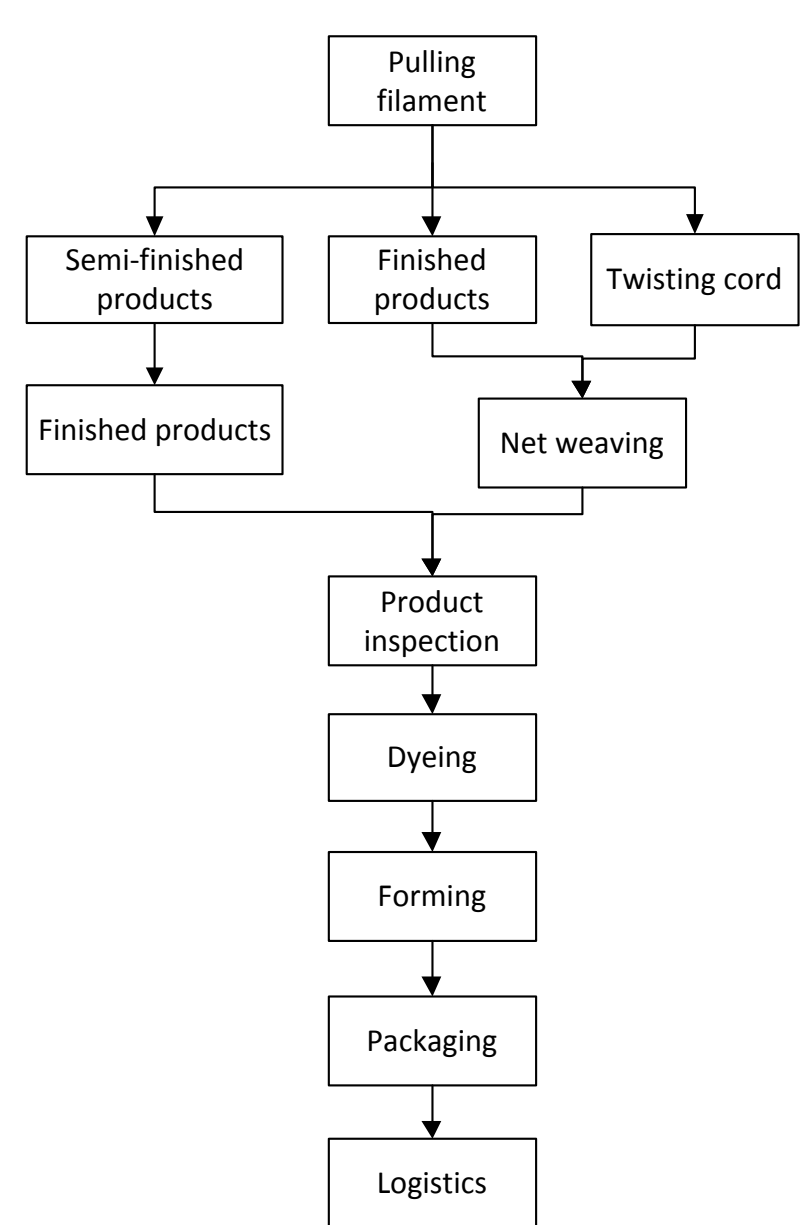

Figure 12. Fishing equipment industry production flowchart.

This paper expects that the smaller the objective function value the better. The various assessment values are defined as follows [4, 24]:

(1) "Number of work stoppages": Number of days off in the plant calendar; fewer days off result in a better assessment value.

(2) "Number of delays": The number of times work on a work piece is delayed; fewer delays result in a better assessment value.

(3) "Maximum delay time": Maximum delay time for all work pieces; shorter delay times result in a better assessment value.

(4) "Mold change cost": The cost of changing a mold on the machine; lower costs result in a better assessment value.

(5) "Processing cost": Work piece processing cost; lower costs result in a better assessment value.

The chromosome objective function value is consequently as follows:

$$
\min Z, \text { with } Z=\sum_{i=1}^{5} w_{i} f_{i}(X), \text { s.t. } X \in \beta
$$

Where

$X$ : work pieces,

$\beta$ : total number of work pieces, $w$ : weighting

$f_{1}(x)=$ number of work stoppages,

$f_{2}(X)=$ number of delays,

$f_{3}(X)=$ maximum delay time,

$f_{4}(x)=$ mold change cost,

$f_{5}(X)=$ processing cost.

\subsection{Fitness design}

The fitness obtained from the fitness function is defined as a function of the objective function $Z$ in the previous subsection, which can be expressed as the following:

$$
\text { fitness }=\frac{1}{Z+1}=\frac{1}{\sum_{i=1}^{5} w_{i} f_{i}(X)+1}
$$

\subsection{Results and discussion}

This case employed a production scheduling system developed using intelligent evolutionary algorithm technology. This system was developed into an application software package integrated computation programs and input/output data via Microsoft Excel. Dynamic information needed by the fishing gear industry was displayed in the form of a Gantt Chart.

The following is a summary of the system model's detailed operating steps:

(1) Customer orders: Filament quantities needed by customers, specifications, and other relevant information is as shown in Figure 13.

(2) Machine capacity and specifications: A specifications table (see Figure 14) was compiled on the basis of factory machine characteristics.

(3) Plant calendar: Machine stop work times and reasons (Figure 15).

(4) Calculation of scheduling: Input necessary data fields based on the foregoing three points; this allows "GA scheduling calculations" to be performed (Figure 16).

(5) Inspection of scheduling results: The results of optimized production scheduling for the fishing gear industry are as shown in Figure 17.

(6) Inspection of Gantt chart: In the Gantt chart showing scheduling results (Figure 18); blue is machine maintenance time and yellow is materials processing time. 


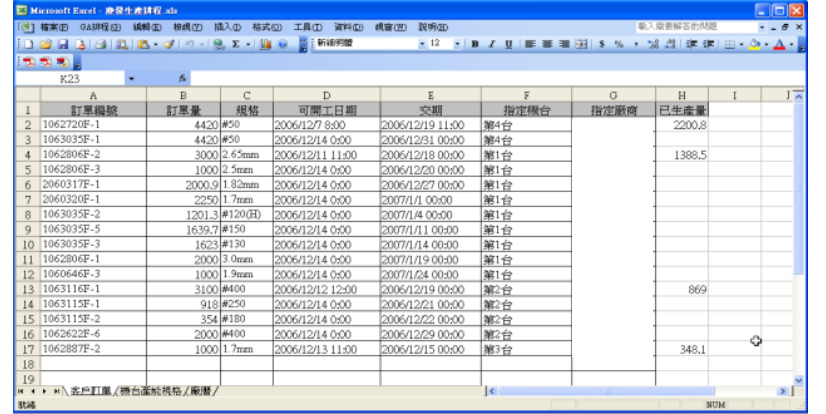

Figure 13. Initialization data - customer orders.

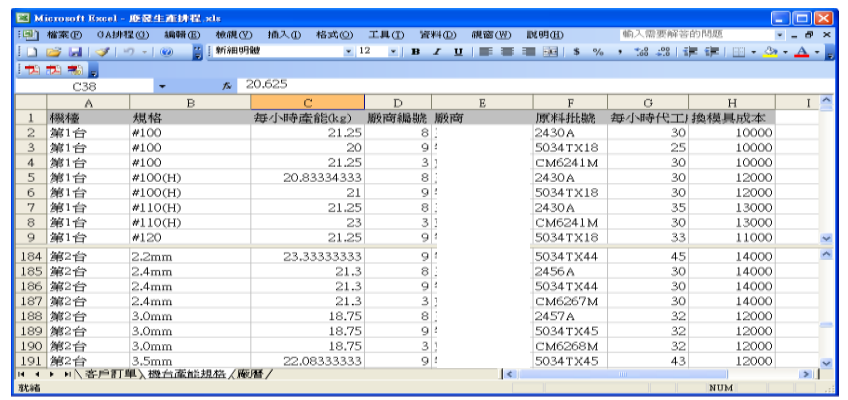

Figure 14. Initialization data - Machine capacity and specifications.

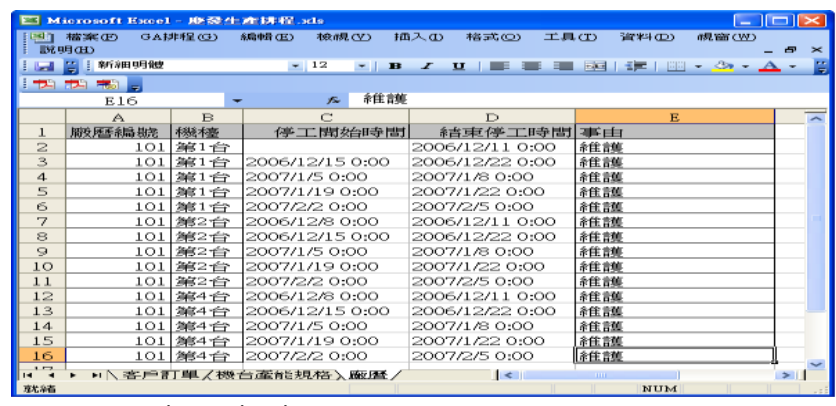

Figure 15. Plant calendar.

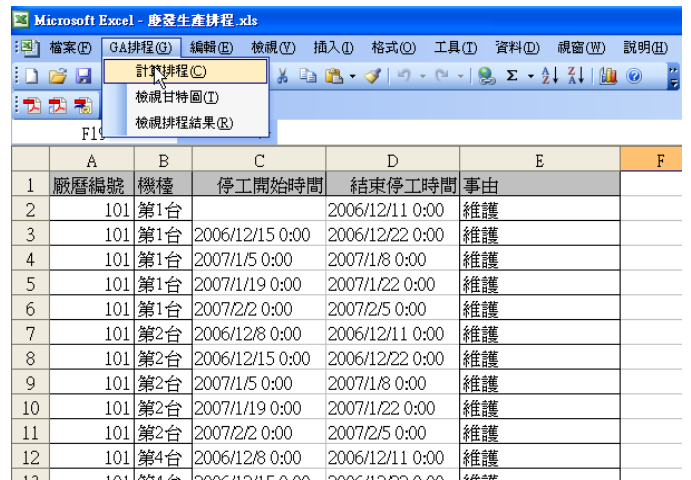

Figure 16. GA scheduling calculations.

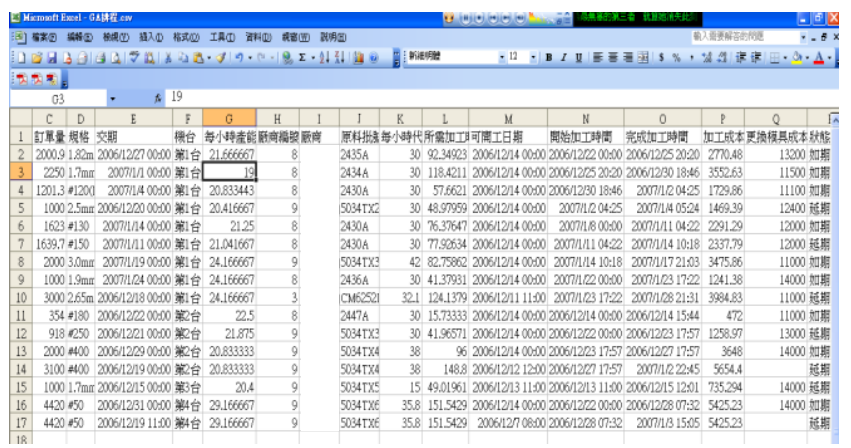

Figure 17. optimization production scheduling results.

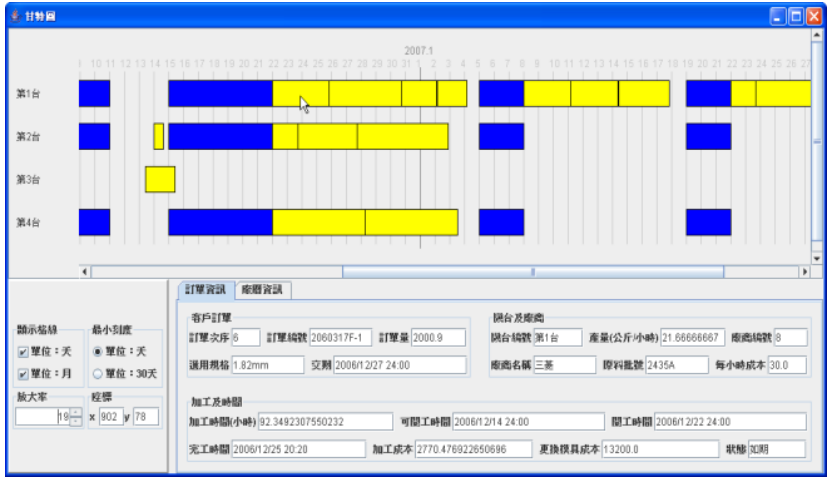

Figure 18. Gantt chart.
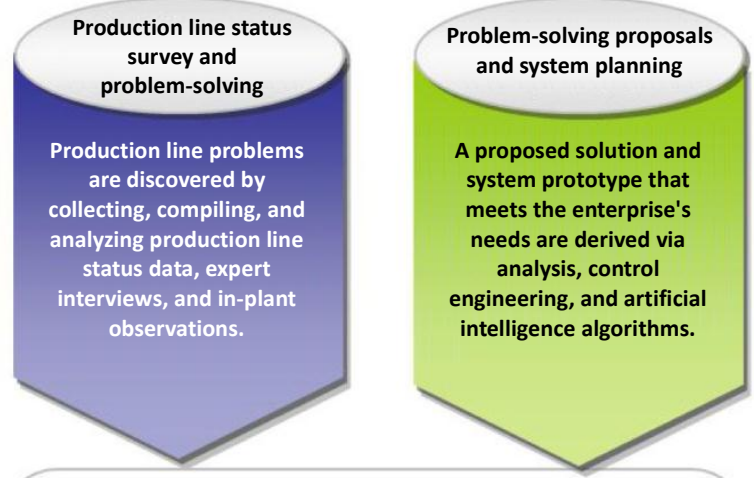

Optimized production process system

Figure 19. Optimal production process system.

\subsection{Contribution}

(1) By applying a genetic algorithm to the production scheduling in the fishing equipment industry, this study effectively overcame the deficiencies of other existing production scheduling methods, reduced the number of machine stoppages, lessened production preparation costs, and reduced order delays and maximum delay time.

(2) By providing the fishing equipment industry with a practical method of obtaining optimal production scheduling solutions, this method will help the industry solve its complex production scheduling problems.

\section{Dynamic real-time production scheduling system design case}

This case involved the use of artificial intelligence in conjunction with a patented genetic algorithm to establish a systematic management method capable of finding blind spots and problems in production processes via two-stage analysis and planning. This system can determine optimal production processes for companies, boost performance and efficiency, and make optimal use of human resources (Figure 19). 


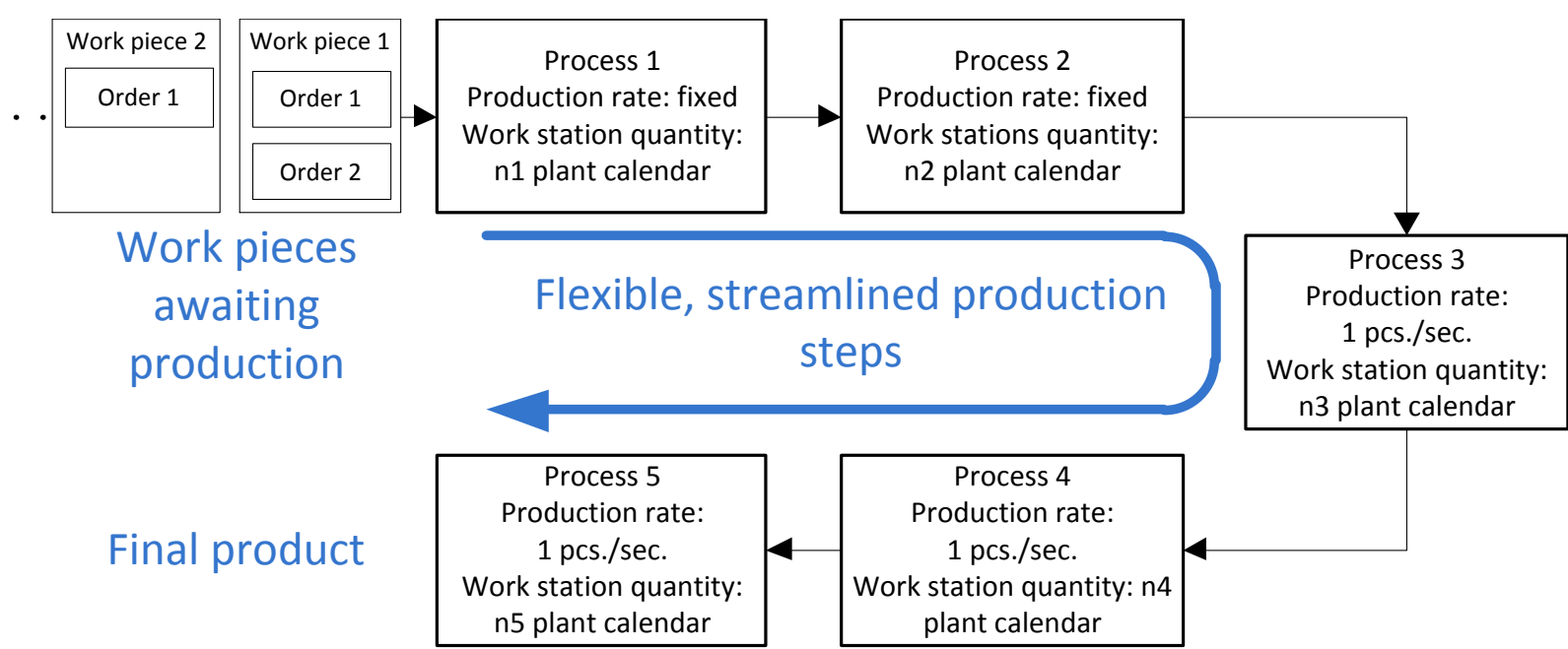

Figure 20. Schematic diagram of five-level processing steps.

As shown in Figure 20, the goals of this case include:

(1) To propose rules for an objective optimization algorithm for metal processing company scheduling, and plan a processing sequence for work pieces awaiting processing.

(2) To write an experimental scheduling system based on the foregoing algorithmic rules, and use this system to verify scheduling performance.

(3) To derive a detailed time schedule for each machine.

\subsection{Chromosome design}

Chromosome design sought to encode flexible production streamlining problems. Each gene represented a work piece, and the locations of genes represented the order of machine resource use. As a result, each chromosome expressed a certain feasible scheduling solution (Figure 21).

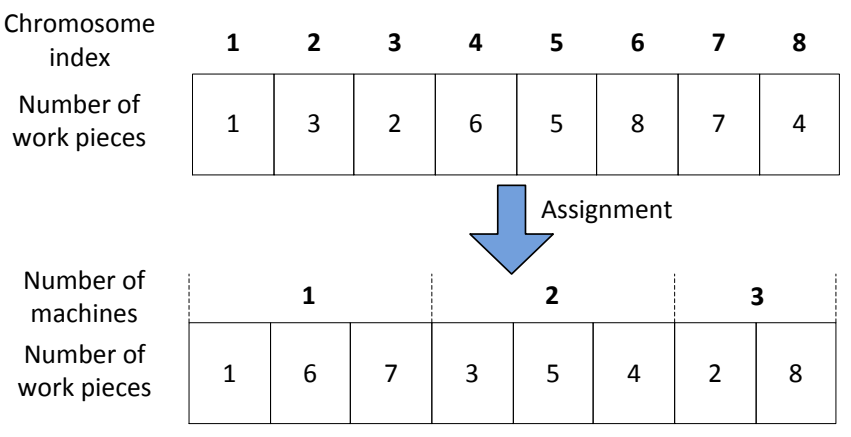

Figure 21. Schematic diagram of chromosome design method.

\subsection{Fitness design}

The fitness function was defined as shown in Equation (10), and was a standard multiobjective function.

$$
\min \Lambda\left(v_{1}, v_{2}, v_{3}\right), \text { where } v_{1}=T S_{N}, v_{2}=T_{N}, v_{3}=T_{M}
$$

\subsection{Results and Discussions}

Infrared and information technology was used to construct a prototype on-site real-time work reporting system. The structure of the prototype dynamic production system is shown in Figure 22 (the dotted lines represent expandable functions).

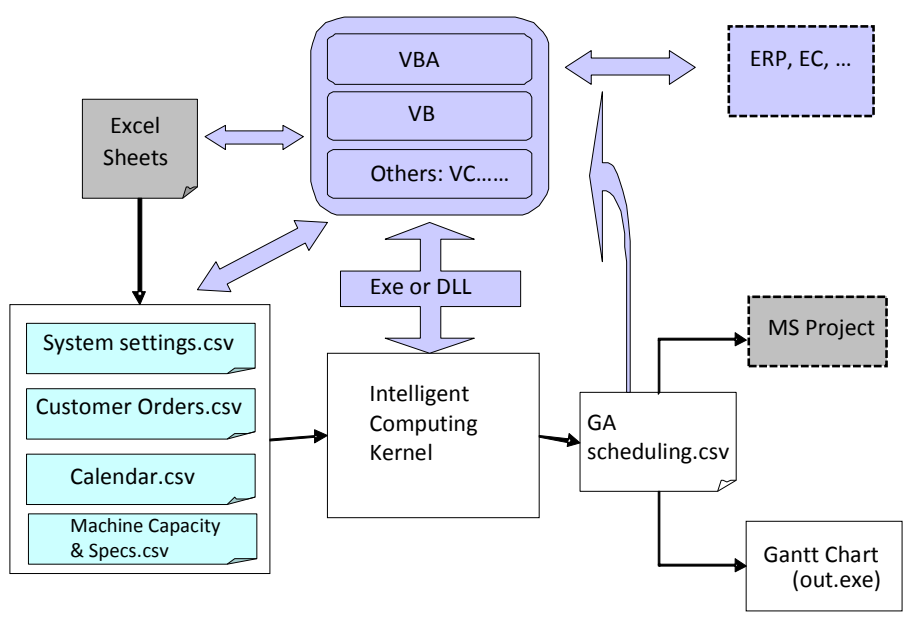

Figure 22. Schematic diagram of prototype dynamic scheduling system. 


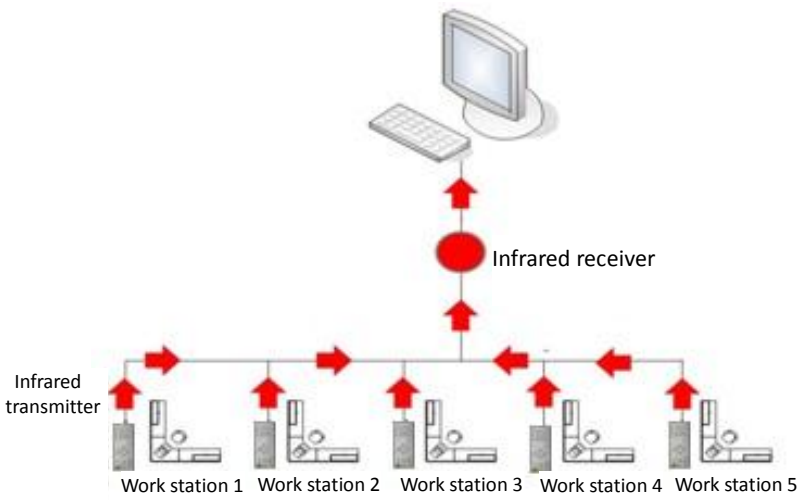

Figure 23. Schematic diagram of infrared on-site real-time work reporting system.

After retrieving Excel data files from existing forms or databases, the system uses programs written in VBA, $\mathrm{VB}$, or $\mathrm{C}$ languages to convert the data-which may include system settings, customer orders, plant calendars, and machine capacity and specifications-to .csv text files. This data is then entered into the genetic algorithm so that .exe or .dll core optimization calculation programs may be written. One advantage of this is that a company does not need to purchase extra software or pay additional employee training costs.

An infrared remote control installed on each machine completed the on-site real-time work reporting system. This system relayed the status of every operating machine to a central computer; by reporting current status and output, the system provides real data enabling a management center to manage production lines, effectively reducing management manpower and equipment costs (Figure 23).

\subsection{Contribution}

(1) It was found that the use of an intelligent evolutionary algorithm technology to determine scheduling order in a production scheduling system can sharply lessen conventional manual scheduling time and reduce the error rate while dramatically shortening scheduling time.

(2) The digitization of customer order specifications can reduce confusion and errors resulting from handwritten order information, and health management personnel to effectively manage order information and query and track production status.

\section{Conclusions and Future Directions}

This paper has proposed applications of intelligent evolutionary algorithm technology to optimal automation system design, and successfully applied this technology to groundbreaking interdisciplinary integrated optimal automation system design. It was found that the approach can resolve long-standing linkage mechanism and production scheduling problems and bottlenecks encountered by relevant industries. Conventional production methods mostly consider cost and performance, and require a sufficient number of lots and experts' precise calculations to ensure successful production. However, in the wake of greater market diversification and manpower shortages, conventional factories facing optimal design problems frequently find that manpower, time, and cost issues prevent them from obtaining optimal solutions, which sometimes even leads to the embarrassing problem of suffering greater losses when more orders are received. If these firms can adopt the intelligent evolutionary algorithm technology proposed in this paper, they may be able to greatly increase their competitive advantage. In the future, we will seek to improve optimized station methods to account for variables and uncertainties involving tolerance design, raw materials, machinery, and manpower, ensuring that automated systems have robustness as well as optimal characteristics, and can better meet the actual needs of industry.

\section{References}

[1] Y. H. Juan, "Application of simulation and genetic algorithm to the identical machine scheduling problem," M.S. thesis, Department of Industrial Engineering and Management, Yuan Ze University, Taiwan, 2002.

[2] T. K. Liu, Production control information system course lecture notes. Taiwan: Department of Mechanical Engineering, National Kaohsiung First University of Science and Technology, 2007.

[3] T. Gen'ichi, C. Subir, and T. Shin, Robust engineering. New York: McGraw-Hill, 2000.

[4] T. K. Liu, J. T. Tsai, and J. H. Chou, "Improved genetic algorithm for the job-shop scheduling problem," The International Journal of Advanced Manufacturing Technology, vol. 27, no. 9-10, pp. 1021-1029, 2005.

doi: $10.1007 / \mathrm{s} 00170-004-2283-4$

[5] J. T. Tsai, T. K. Liu, and J. H. Chou, "Hybrid taguchi-genetic algorithm for global numerical optimization," IEEE Transactions on Evolutionary Computation, vol. 8, no. 4, pp. 365-377, 2004. doi: $10.1109 /$ TEVC.2004.826895

[6] J. H. Chou, W. H. Liao, and J. J. Li, "Application of Taguchi-genetic method to design optimal grey-fuzzy controller of a constant turning force system," in $15^{\text {th }}$ Annual Conference of the Chinese Society of Mechanical Engineers, Taiwan, 1998, pp. 31-38. 
[7] Y. Tsujimura, Y. Mafune, and M. Gen, "Effects of symbiotic evolution in genetic algorithms for job-shop scheduling," in, $34^{\text {th }}$ Annual Hawaii International Conference on System Sciences, Los Alamitos, CA, USA, 2001, pp. 3026-3026. doi: 10.1109/HICSS.2001.926320

[8] M. Gen and R. Cheng, Genetic algorithms and engineering design, $1^{\text {st }}$ ed. New York: Wiley-Interscience, 1997.

[9] Y. M. Kuo, "Applying niche genetic algorithms to optimizing motion control of multi-DOF robots," M.S. thesis, Graduate Institute of Mechanical and Automation Engineering, National Kaohsiung First University of Science and Technology, 2008.

[10] G. Shl, "A genetic algorithm applied to a classic job-shop scheduling problem," International Journal of Systems Science, vol. 28, no. 1, pp. 25-32, 1997.

doi: $10.1080 / 00207729708929359$

[11] J. H. Chou, Experimental design and quality engineering lecture notes. Taiwan: Graduate Institute of Systems and Control, National Kaohsiung First University of Science and Technology, 2005.

[12] K. J. Waldron and G. L. Kinzel, Kinematics, dynamics, and design of machinery, $2^{\text {nd }}$ ed. New York: Wiley, 2004.

[13] R. Norton, Fundamentals of machine design. New York: McGraw-Hill, 2004.

[14] M. A. Laribi, A. Mlika, L. Romdhane, and S. Zeghloul, "A combined genetic algorithm-fuzzy logic method (GA-FL) in mechanisms synthesis," Mechanism and Machine Theory, vol. 39, no. 7, pp. 717-735, 2004. doi: $10.1016 /$ j.mechmachtheory.2004.02.004

[15] I. M. Huang, "Intelligent design of six-bar linkage mechanisms using multi-objective genetic algorithms," M.S. thesis, Graduate Institute of Mechanical and Automation Engineering, National Kaohsiung First University of Science and Technology, 2009.

[16] H. Zhou, "Dimensional synthesis of adjustable path generation linkages using the optimal slider adjustment," Mechanism and Machine Theory, vol. 44, no. 10, pp. 1866-1876, 2009. doi: $\underline{10.1016 / j . m e c h m a c h t h e o r y .2009 .03 .010}$
[17] D. Y. Dai, "Optimal design of multi-modal six-bar linkage mechanism using niche genetic algorithms," M.S. thesis, Graduate Institute of Mechanical and Automation Engineering, National Kaohsiung First University of Science and Technology, 2009.

[18] X. Yu and B. Meng, "Research on dynamics in group decision support systems based on multi-objective genetic algorithms," in International Conference on Service Systems and Service Management, Troyes, France, 2006, pp. 895-900. doi: $10.1109 /$ ICSSSM.2006.320750

[19] X. Hu and C. Xie, "Niche genetic algorithm for robot path planning," in Third International Conference on Natural Computation (ICNC), Haikou, China, 2007, pp. 774-778. doi: 10.1109/ICNC.2007.502

[20] Y. L. Zheng and D. M. Lei, "Hybrid niche genetic algorithm for set covering problem," in, International Conference on Machine Learning and Cybernetics, Hong Kong, 2007, pp. 1009-1013. doi: 10.1109/ICMLC.2007.4370290

[21] B. Huang, Z. Wang, and Y. Xu, "Multi-objective genetic algorithm for hybrid electric vehicle parameter optimization,"in International Conference on Intelligent Robots and Systems, Beijing, China, 2006, pp. 5177-5182. doi: $10.1109 /$ IROS.2006.281654

[22] Y. Tsujimura, M. Gen, and R. Cheng, "Improved genetic algorithms for solving job-shop scheduling problem," Engineering Design and Automation, vol. 3, no. pp. 133-144, 1997.

[23] Y. C. Chen, "Applications of multi-objective genetic algorithms for real-world optimization in production scheduling," M.S. thesis, Graduate Institute of Mechanical and Automation Engineering, National Kaohsiung First University of Science and Technology, 2008.

[24] T. K. Liu, J. T. Tsai, J. H. Chou, and C. H. Lai, "Job-shop scheduling problems by using an improved genetic algorithm," in Annual Conference of the Society of Instrument and Control Engineers (SCIE), Okayama, Japan, 2005, pp. 944-949. 